\title{
Optimization of the electric vehicle HVAC duct system based on gradient method
}

\author{
A. Maksimov ${ }^{1}$, D. Igoshina, R. Petrov, O. Klyavin \\ Peter the Great St. Petersburg Polytechnic University \\ Russia
}

Received: June 18, 2020. Revised: July 22, 2020. Accepted: July 24, 2020. Published: July 27, 2020.

\begin{abstract}
Our study describes the optimization of the part of heating, ventilation and air conditioning (HVAC) system of an electric car. The geometry of the air duct connected to the heater unit on one side and the dashboard on the other. From the point of view of aerodynamics, it is necessary to change the geometry of the air duct so as to minimize the loss of air pressure and ensure a more uniform flow from the exit holes to improve the efficiency of windshield heating. In order to optimize the initial geometry of the air duct, approach of using gradient methods was applied. According to the results of the study, detailed concept of optimizing the air duct of an electric car is presented. The optimized geometry was tested with the use of methods of computational aerodynamics. Calculations were made as part of the interior model of electric car with the output of temperature fields on the windshield. The results can be applied to the design of heating, ventilation and air conditioning systems of vehicles in a short time.
\end{abstract}

Keywords-Adjoint solver, air duct, electric vehicle, windshield heating.

\section{INTRODUCTION}

$\mathrm{O}$ ptimizing the shape of air ducts is a powerful technical tool in the design of vehicle ventilation systems. Parametric optimization as a means of finding the best design when analyzing a large number of geometries of the investigated part, calculations and comparison of results is a very expensive method in terms of computer and engineering time. Parametric optimization is limited by the original CAD (computer aided design) geometry, in which we can change only the basic geometric parameters, such as lengths, angles, radiuses. In order to improve the efficiency of the optimization process, an optimization algorithm based on changing the

\footnotetext{
${ }^{1}$ Corresponding author: maksimov@compmechlab.ru
}

The work was performed with financial support of the Ministry of Science and Higher Education of the Russian Federation as part of the implementation of the Federal Target Program "Research and development in priority areas of the scientific and technological complex of Russia for 2014-2020". Unique identifier of the agreement RFMEFI57818X0269. shape of the finite element mesh has been developed, which allows you to preserve the quality of design and reduce the time from the first developed geometry to the optimized form.

Optimization includes all aspects of the design process as well as the control of pressure and velocity fields at the air inlet and outlet [1], [2]. Therefore, a modern approach to optimization has been chosen, where it is possible to change the geometry locally, using the adjoint method to optimize the designed duct of electric car.

The adjoint method is an iterative numerical method for solving optimization problems, which allows to determine the extremums of the target function, is one of the gradient methods of optimization. This gradient method is able to calculate the sensitivity of the target function to the optimized parameters. It is proved that the adjoint method exceeds other methods described, for example, as in [3].

Similar research in this area is carried out by the Department of Applied Mechanics at the Chalmers University of Technology in Göteborg, Sweden. In this paper the author gives an assessment of the usability, reliability and simplicity of the adjoint solver in Ansys Fluent [4].

The main objectives of this study are: optimization of the air duct by the adjoint method for achieving thermal efficiency when heating the windscreen, comparison of the results of parametric optimization and optimization by the adjoint method.

Our research is based on aerodynamic optimization in Ansys Fluent software. The target functions are the reduction of pressure differences in the duct and the uniform distribution of the velocity field at the air duct outlet. Due to the complex geometry and losses in the air ducts, the maximum thermal efficiency required for flow on the windscreen of electric vehicle is achieved with a uniform flow at the outlets. For the subsequent works related to the overall optimization of the ventilation, heating and air conditioning system of the passenger car, we have received an optimized and heat efficient CAD model of the duct for heating the windscreen.

\section{MATERIALS AND METHODS}

\subsection{Case Study and Numerical Procedure}

The stationary viscous incompressible gas flow describes the Navier-Stokes equation (1), which is the basic system of gas dynamics equation: 


$$
\frac{d(\rho \underline{\vec{V}})}{d t}+\nabla \cdot(\rho \underline{\vec{V} \vec{V}})=-\nabla\left(P_{e f f}\right)+\nabla \cdot\left[2\left(\mu_{e f f}\right) \bar{S}\right]
$$

where $\rho$ - density, $V$ - speed, $P_{\text {eff }}$ and $\mu_{\text {eff }}$ - effective pressure and viscosity, $S$ - strain rate tensor.

The turbulence model $k-\varepsilon$ realizable was used to close the system of equations (1). In comparison with the standard $k-\varepsilon$ model, this model has a different formulation of turbulent viscosity, and the transfer equation for dissipation rate $\varepsilon$ has been changed [5].

To obtain the values of the kinetic energy of turbulence $k$ and the rate of its dissipation $\varepsilon$, the transfer equations (2) and (3) are solved for the characteristics, which can be obtained by expressing the turbulent viscosity $\mu_{t}[6]$ :

$\frac{d}{d t}(\rho k)+\frac{d}{d x_{i}}\left(\rho k u_{i}\right)=\frac{d}{d x_{j}}\left[\left(\mu+\frac{\mu_{t}}{\sigma_{k}}\right) \frac{d k}{d x_{j}}\right]+G_{k}+G_{b}-\rho \varepsilon-$

$Y_{M}+S_{k}$

$\frac{d}{d t}(\rho \varepsilon)+\frac{d}{d x_{i}}\left(\rho \varepsilon u_{i}\right)=\frac{d}{d x_{j}}\left[\left(\mu+\frac{\mu_{t}}{\sigma_{\varepsilon}}\right) \frac{d \varepsilon}{d x_{j}}\right]+C_{1 \varepsilon} \frac{\varepsilon}{k}\left(G_{k}+\right.$ $\left.C_{3 \varepsilon} G_{b}\right)-C_{2 \varepsilon} \rho \frac{\varepsilon^{2}}{k}+S_{\varepsilon}$

where $G_{k}$ is responsible for "generating" the kinetic energy of turbulence associated with the average speed gradient, $G_{b}$ responsible for "generating" the kinetic energy of turbulence associated with the ejection force, $Y_{M}$ - represents the contribution of fluctuating dilatation (in the turbulence of the compressible liquid) to the total value of dissipation; $C_{1 \varepsilon}, C_{2 \varepsilon}$, $C_{3 \varepsilon}$ - constants; $\sigma_{k}$ and $\sigma_{\varepsilon}$ - Prandtl turbulent numbers for $k$ and $\varepsilon$, respectively; $S_{k}$ and $S_{\varepsilon}$ - given initial conditions; $\varepsilon$ dissipation.

Turbulent viscosity in the proposed model is written in the following form, equation (4) [7]:

$$
\mu_{t}=\rho C_{\mu} \frac{k^{2}}{\varepsilon}
$$

where $\rho$ - density, $k$ - kinetic energy of turbulence, $\varepsilon$ - rate of its dissipation, $C_{\mu}$ - a function of the mean strain and rotation rates.

The problem described by the Navier-Stokes equations for the stationary gas flow in Ansys Fluent is solved by the finitevolume method, the pressure-velocity relationship is established by the SIMPLEC (Semi-Implicit Method for Pressure Linked Equations - Consistent) algorithm.

The target function is pressure drop minimization (a linear combination of pressure minimization and volume integral velocity at the air duct outlet) [8].

To output the target function, it is necessary to solve the related equations (5):

$$
\frac{\partial d}{\partial \varphi}=\propto \frac{\partial R}{\partial \varphi}
$$

where $\propto$ - the Lagrange multiplier, $\varphi$ - flow state, $d$ - the desired function, $R$ - residual.

The adjoint equation (5) needs to be solved once to obtain the sensitivity vector of the structure with respect to design variables (6):

$$
\delta d=\left(\frac{\partial d}{\partial c}-\alpha \frac{\partial R}{\partial c}\right) \delta c
$$

where $d$ - the desired function, $c$ - the controlled variable, $\alpha$ - the Lagrange multiplier, $R$ - residual.

Once the sensitivity values of the surface are obtained, the finite-volume mesh will deform.

The adjoint equations are solved using the adapted SIMPLEC algorithm [9], [10].

Fig. 1 shows the SIMPLE algorithm, where $p^{*}$-guessed pressure, $u^{*}, v^{*}$-velocities based on $p^{*}, p^{\prime}$-pressure correction, $p$-pressure, $u, v$-velocity components in $\mathrm{x}, \mathrm{y}-$ directions.

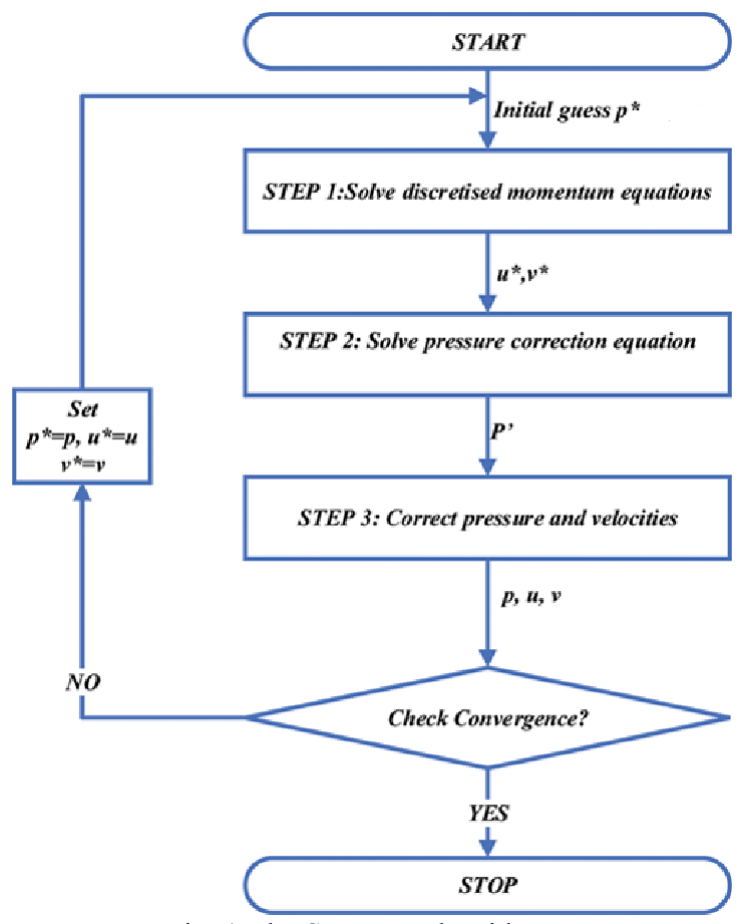

Fig. 1 The SIMPLE algorithm

The SIMPLEC algorithm is almost same as the SIMPLE algorithm, except that the manipulations are changed to ensure lesser omissions (it allows SIMPLEC velocity correction equations to omit terms that are less significant than those omitted in SIMPLE). With SIMPLEC, the pressure-correction under-relaxation factor is generally set to 1.0 , which aids in convergence speed-up. The authors of [11], [12] say that the SIMPLEC method beats other methods (SIMPLER (SIMPLE - Revised), SIMPLE) in the amount of time spent by the processor on 1 iteration of the calculation. On average, the SIMPLEC method is 1.2-1.3 times faster than SIMPLE.

After selecting the optimization method to change the air duct shape and minimize air flow losses, the model is 
unloaded into Ansys Fluent. Fig. 2 shows the work of the optimization algorithm as a block diagram.

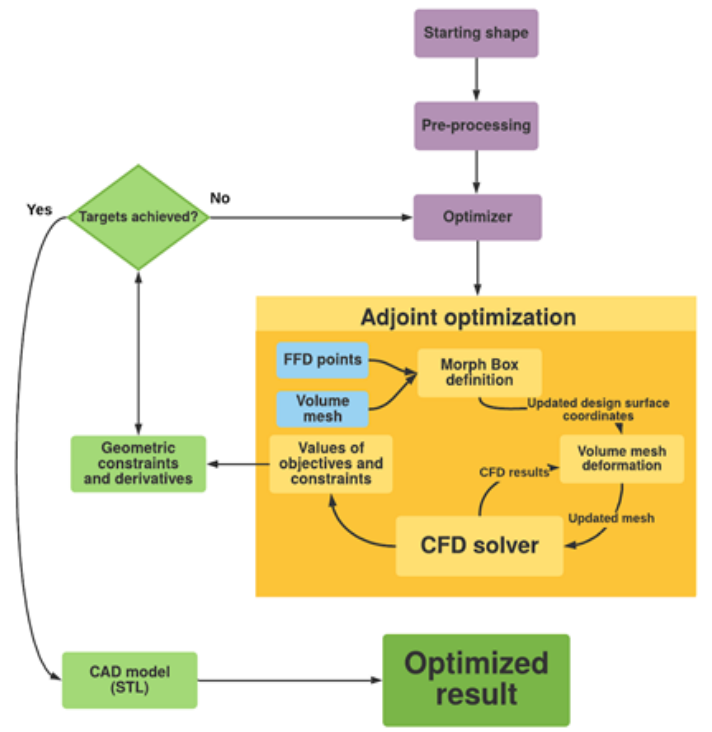

Fig. 2 Block diagram of the vehicle air duct optimization

The program generates a finite-volume mesh with prism layers and sets boundary and initial conditions (Fig. 3).
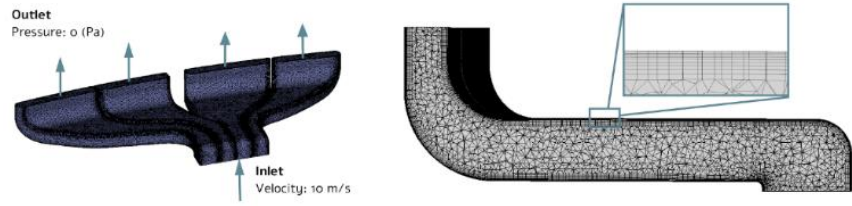

Fig. 3 A finite-volume mesh of duct with the specified boundary conditions

The boundary prism layers is created in a finite-volume mesh model near the surface of solid walls. On these impermeable walls, the boundary condition without slippage is applied, the normal and tangential speed components are zero.

At the inlet, the flow speed and temperature are set. In our work, flow rate and temperature were the values measured experimentally from a prototype heater. The outlet opening of the heater in turn connects to the inlet opening of the duct without additional connecting tubes. At the outlet, a zero overpressure is set. Air is used as a design medium with a specific heat capacity of $1004.4 \mathrm{~J} / \mathrm{kg} \cdot \mathrm{K}$, thermal conductivity of $0.0261 \mathrm{~W} / \mathrm{m} \cdot \mathrm{K}$, viscosity of $1.831 \cdot 10^{-5} \mathrm{~kg} / \mathrm{m} \cdot \mathrm{s}$ and density calculated according to an ideal gas law. Despite the fact that the air flow rate from the heater is much lower than the number of Mach $<0.3$ and it is logical to use gas with a constant density, it was decided to use the law of ideal gas because of the temperature difference in the interior of the electric car and the air flow from the heater unit.

\subsection{The Adjoint solver}

The Adjoint Solver module in Ansys Fluent is a tool to optimize geometric models used in CFD (computational fluid dynamics) calculations. The interface of the adjoint control settings is shown in Fig. 4. Setting up the solver allows to specify the advancement controls and stabilization schemes.
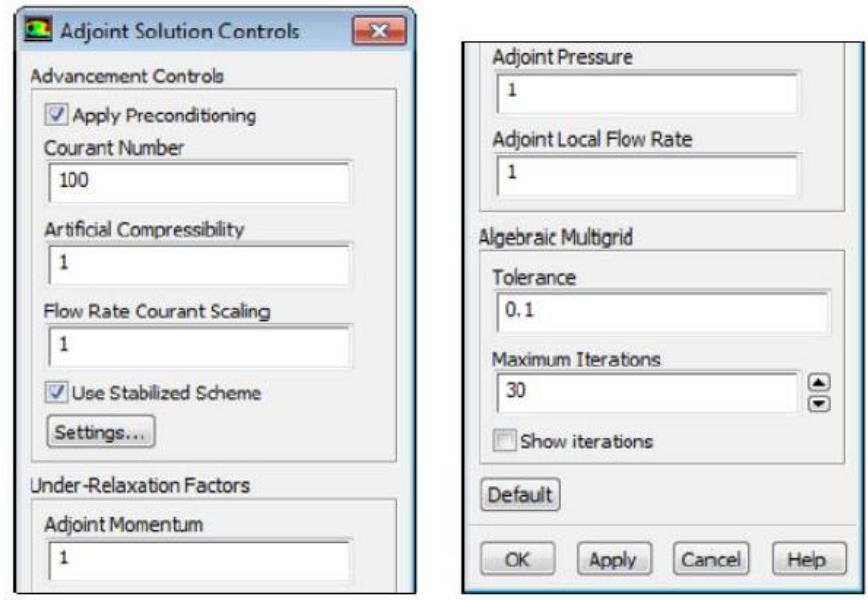

Fig. 4 Interface of the adjoint solution controls

According to the recommendation [4], the solver was set up as follows:

1) The pressure-velocity coupling was computed by SIMPLEC scheme.

2) The gradient was computed by Green-Gauss Cell-Based Gradient scheme.

3) The momentum, turbulent kinetic energy, turbulent dissipation rate were represent by Second Order Upwind scheme.

In addition, the advancement controls can be specified:

1) The Courant Number is the dimensionless number that is used in the Courant-Fredrichs-Lewy condition. For our solver $C_{\text {max }}>1$

2) The artificial compressibility method helps to overcome the difficulty of the pressure decoupling, $\beta \leq 1-$ the artificial compressibility.

3) Flow rate courant scaling affects on the stability of $\mathrm{AMG}$ solver (default value is 1 , lower is better).

4) Under relaxation factors (URF) for adjoint pressure/ momentum/ local flow rate were set on 0.6 by default. Higher value shows to higher contribution of new solution to old one which might lead to solver instabilities.

5) Algebraic multigrid. The tolerance of convergence and the Maximum Iterations for inner Algebraic Multi Grid (AMG) iterations can be set.

Fig. 5 shows the mesh model of the air duct to be optimized and the area of optimization as a rectangle. Input and output holes lie outside the optimization area, their geometry is fixed and cannot be changed. 


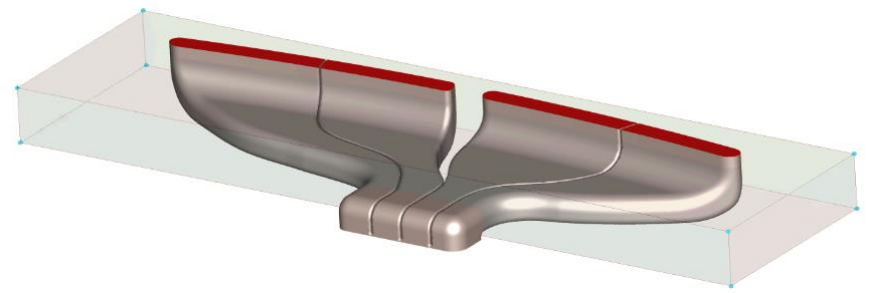

Fig. 5 Location of the deformation zone

Using the mesh deformation technology, an area is built around the model to change the original finite volume mesh model. We can change the location and size of the morphing area during the aerodynamic optimization process - in this way we can manage areas where different degrees of mesh model changes are required. The authors of [4] note that when working with Ansys Control Volume Morphing you should be attentive to the parameters of the mesh, because, for example, distorted cells can give negative volume.

The final duct geometry changes smoothly depending on the number of attempts to change the target values. After each iteration, the new geometry is available for consideration. A process engineer who knows the manufacturing process for this type of part can evaluate whether this design is suitable for real production by vacuum forming or not.

The final check of the optimized model is that we do aerodynamic calculations using a full model of the interior of the electric car. Fig. 6 shows the front part of the geometry of an electric car with boundary conditions.

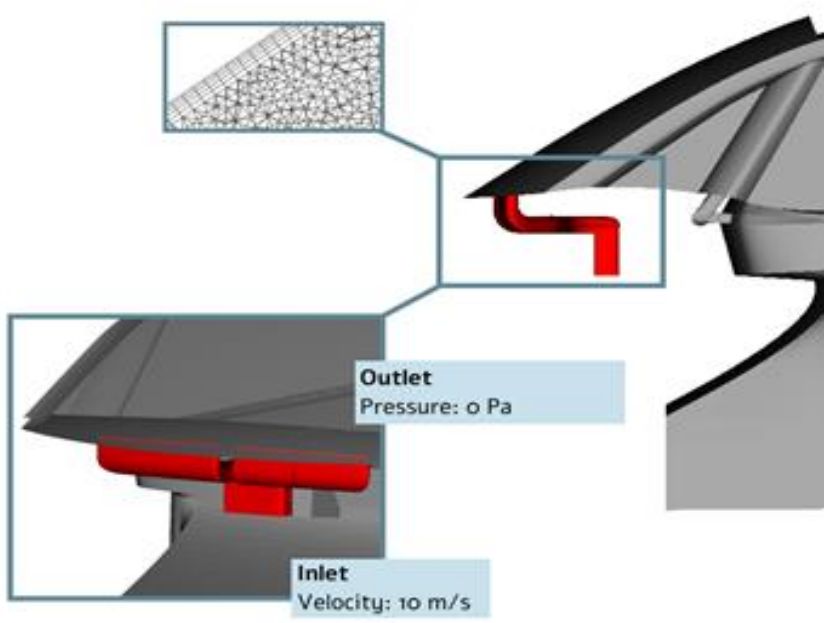

Fig. 6 Geometry of the interior of electric car

As the initial conditions, the velocity $10 \mathrm{~m} / \mathrm{s}$ and the initial temperature $15^{\circ} \mathrm{C}$ were set.

\section{RESULTS AND DISCUSSION}

Fig. 7 shows the result of optimization of the air duct interior space.

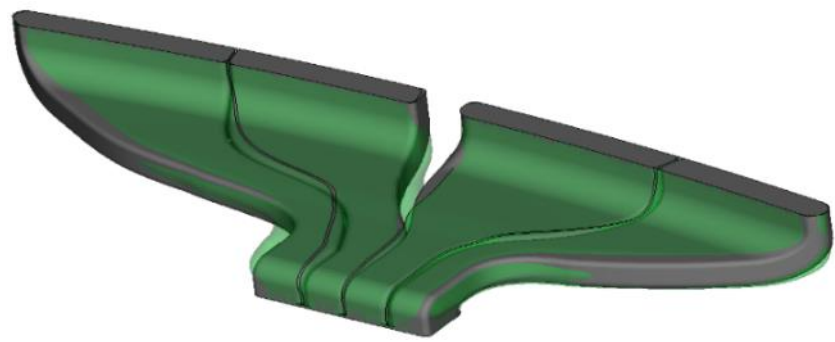

Fig. 7 Result of optimization of the air duct geometry (grey initial geometry, green - optimized)

As the target value should be changed smoothly to avoid negative volumes in the mesh model, for this geometry of the air duct the result with the number of iterations $\mathrm{N}=7$ is shown in Fig. 7 and Fig. 8. Fig. 8 shows the velocity fields near the impermeable walls of initial and optimized geometry. We can see that the velocity field at the outlet of the optimized geometry has become more homogeneous, while the duct geometry has changed slightly.

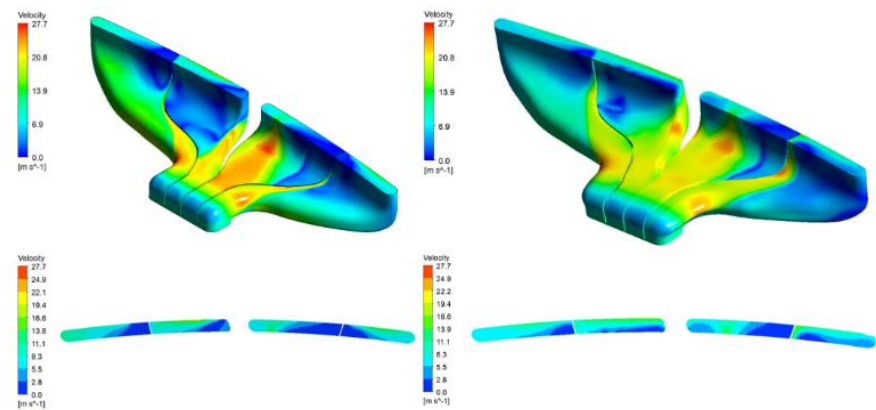

Fig. 8 Result of optimization of the air duct (initial geometry on the left, optimized on the right)

As a result of aerodynamic optimization, it was possible to create more homogeneous flow at the outlet of the air duct and to minimize pressure loss by $24 \%$.

After optimization the mesh model should be processed in CAD software to make a solid-state model its subsequent transfer on manufacture and creation of the design documentation. In addition, it is necessary to perform an aerodynamic calculation of the resulting optimized duct in the interior of the car with the determination of temperature fields on the windshield.

The result of the above-mentioned optimization method is a drop of $11 \%$ in the value of the target function (pressure difference) for a duct located on the driver's side in the front ventilation grille.

For the initial geometry, the inlet and outlet pressure values were $P_{\text {inl }}=1000 \mathrm{~Pa}, P_{\text {out }}=800 \mathrm{~Pa}$. The pressure difference was $200 \mathrm{~Pa}$.

Due to the optimized geometry, we have reached a pressure drop of $178 \mathrm{~Pa}\left(P_{\text {inl }}=1000 \mathrm{~Pa}, P_{\text {out }}=822 \mathrm{~Pa}\right)$.

Similar work can be considered optimization of car intercooler produced by employees of the Department of Hydromechanics and Hydraulic Equipment, Faculty of Mechanical Engineering, VŠB -Technical University of Ostrava, Czech Republic. As in the above-mentioned work, 
the target function was pressure difference. Eventually, the authors of the work achieved a $23.9 \%$ decrease in the value of the target function [13].

Currently, to optimize the shape of various parts in the automotive industry uses not only the adjoint solver of Ansys, such a solver is available in a set of French company Dassault - Tosca Fluid [14]. Similar work is being done by Ford Europe as part of the Horizon 2020 program, and the result is a $75 \%$ reduction in the pressure differential from the original geometry [15].

The final step is a CFD calculations based on the full model of the car interior. The calculations are transient. Result of the calculations is the temperature fields on the windshield surface. Fig. 9 shows the temperature fields of the windshield of the initial design developed by the design engineer (a), modified by the results of experimental tests (b) and optimized (c) according to the methods presented in this article.

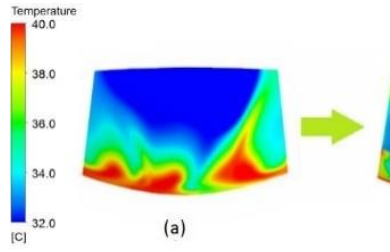

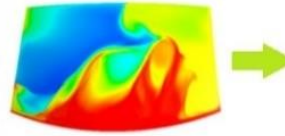

(b)

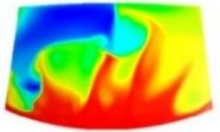

(c)
Fig. 9 Temperature fields of the electric car windshield ((a)original design, (b)-modified, (c)-optimized) at a time of $10 \mathrm{~min}$

Transient calculations were carried out with time step $\mathrm{t}=0.04 \mathrm{~s}$ with a total time of $15 \mathrm{~min}$. The temperature at the air duct inlet was $40^{\circ} \mathrm{C}$ and velocity was $10 \mathrm{~m} / \mathrm{s}$. No-slip boundary conditions were applied on the walls of the interior of the electric car. The model of turbulence used in the calculation - Detached Eddy Simulation. The number of elements in the finite-volume mesh of the interior is about 45 million (Fig. 10).

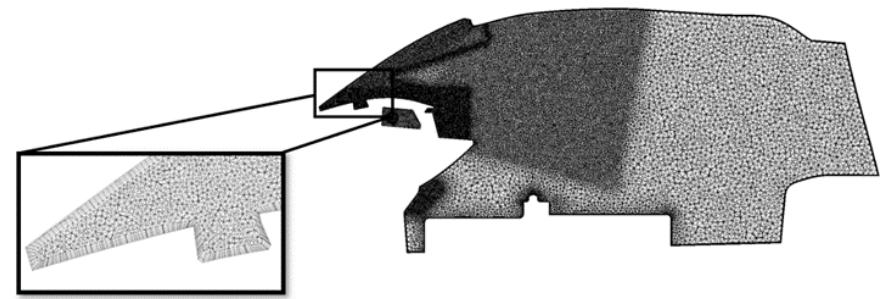

Fig. 10 Final-volume mesh of the electric vehicle interior used for transient simulation

It can be clearly seen that the temperature fields for the initial design of the duct (a) do not warm well the central area of the windscreen (Fig. 9) and go into the driver's outermost area at a sharp angle. The initial design of the duct is unsatisfactory. In the modified (b) (Fig. 11) version of the air duct by CAD-designer, additional flow guides were added. As a result of aerodynamic calculation, a good picture of temperature distribution is obtained (Fig. 9). The flow corresponding to the four duct outlets warm up a wider section of the windscreen than the original air duct. Optimized option (c) shows the best result among the considered options. The driver's area warms up very well to high temperatures, which means that ice and snow will melt quickly.

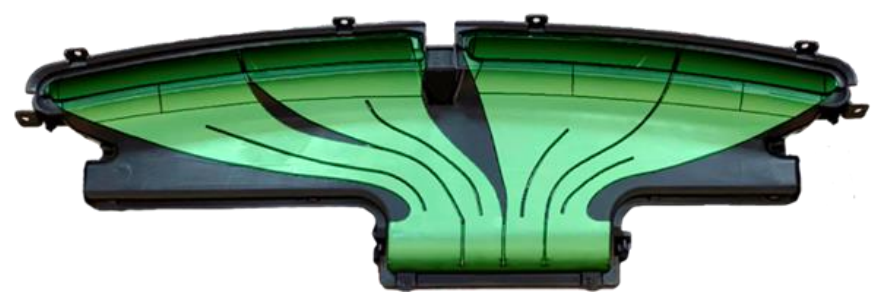

Fig. 11 Manufactured modified prototype (b) of air duct with the shown internal structure

It should be noted that variants (b) and (c) are similar in temperature distribution on the windshield. However, the time spent by an engineer on finishing the construction and obtaining a good temperature distribution on the windshield without using modern simulation technologies is simply not comparable to the time spent on obtaining the option (c). So, for example, to get the variant (c) the engineer spent about 3 days of working time taking into account aerodynamic modelling and creation of the subsequent 3D CAD model. It takes about 2 weeks to create variant (b), which takes into account the time of manufacturing, fine-tuning and remanufacturing of the prototype. The optimized version (c) can be easily obtained by vacuum forming.

As a future study, a solidification/melting model can be considered to simulate multiphase flow taking into account the phase change and flow around electro car.

\section{CONCLUSION}

Optimizing the air duct of the electric car to improve the efficiency of windshield heating was the main goal of this research work. In the course of our work, we have described in detail one of the modern optimization methods - the adjoint method, which in its turn has an advantage in accuracy and speed over the parametric approach to geometry optimization.

We presented initial geometric model of the duct for heating the electric car windshield together with a block diagram of the optimizer. For $\mathrm{N}=7$, where $\mathrm{N}$ is the number of calculated iterations, we optimized the duct for the requirements of GOST 30593-2015.

This document was chosen as the main one since the developed car model with the optimized HVAC system is supposed to be used on the Russian market. It was necessary to satisfy requirements such as temperature and flow rate control, velocity limitations. For example, the flow velocity in the head area should not exceed $0.3 \mathrm{~m} / \mathrm{s}$. In the zone of the belt of the driver and passengers, the flow velocity should be in the range of $0.5-1.5 \mathrm{~m} / \mathrm{s}$.

If it is necessary to provide developments to the international market, it makes sense to also check them according to ISO / ASHRAE Standard.

The prototype was made and its experimental studies were conducted. The prototype is a modified by CAD-engineer the original design. The characteristics of the prototype and the developed optimized air duct were compared using CFD methods. 
During the optimization we managed to reduce the pressure drop by $11 \%$ and create a more homogeneous air flow at the air duct outlet. To confirm that the optimization was successful, we performed a CFD calculation taking into account the interior of the electric car with the output of temperature fields on the windshield.

\section{References}

[1] M. H. Straathof, G. Carpentieri, M. J. L. van Tooren, "Aerodynamic shape optimization using the adjoint Euler equations", Engineering Computations, Vol. 30 No 4, 2013, pp. 469-493.

[2] M. B. Giles, N. A. Pierce, "An introduction to the adjoint approach to design", Flow, turbulence and combustion, Vol. 65 (3-4), 2000, pp. 393-415.

[3] K. C. Giannakoglou, D. I. Papadimitriou, "Adjoint methods for shape optimization", Optimization and computational fluid dynamics, Springer, Berlin, Heidelberg, 2008, pp. 79-108.

[4] A. Tzanakis, "Duct optimization using CFD software ANSYS Fluent Adjoint Solver", Chalmers University of Technology in Göteborg, 2014

[5] F. R. Menter, "Two-equation eddy-viscosity turbulence models for engineering applications", AIAA Journal, Vol. 32 No 8, 1994, pp. 1598-1605.

[6] A. V. Garbaruk, M. Kh. Strelets, M. L. Shur, "Turbulence modeling in of complex flows calculation", Polytechnic Publishing House, SPb, 2012.

[7] A. V. Kuzminov, V. N. Lapin, S. G. Chernyy, "Method for calculating turbulent incompressible fluid flows based on a two-layer (k- $\varepsilon)$ model", Computational Technologies, Vol. 6 No 5, 2001, pp. 73-86.

[8] P. Bradshaw, D. H. Ferriss, N. P. Atwell, "Calculation of boundary-layer development using the turbulent energy equation", Journal of Fluid Mechanics, Vol. 28 No 3, 1967, pp. 593-616.

[9] S. V. Patankar, "Numerical heat transfer and fluid flow", Hemisphere Publishing Corporation, New York, 1980

[10] E. V. Pechenik, "Optimization of the symmetric profile by conjugated gradient method", Izvestia Samara Scientific Center of the Russian Academy of Sciences, Vol. 13 No 1(2), 2011, pp. 330-333.

[11]M. Oriani, G. Pierrot, "Alternative solution algorithms for primal and adjoint incompressible Navier-Stokes", ECCOMAS Congress, 2016, pp. 3858-3882.

[12] J. P. Van Doormall, G. D. Raithby, "Enhancements of the simple method for predicting incompressible fluid flows", Numerical Heat Transfer, Vol. 7, 1984, pp. 147-163

[13] V. Horová, M. Bojko, J. Dobeš, "Methodology of using the Adjoint solver optimization tool during flow in the intercooler filling line to minimize pressure drop", EPJ Web of Conferences, 2019.

[14]M. Stephan, P. Häußler, M. Böhm, "CFD Topology Optimization of Automotive Components ", EASC, 2009.

[15] L. Wendling, M. Behr, A. Hopf, F. Kraemer, "CFD Simulation of Oil Jets for Piston Cooling Applications Comparing the Level Set and the Volume of Fluid Method", SAE Int. J. Adv. \& Curr. Prac. in Mobility 1(2), 2019, pp. 550-561.
[16] Alvaro Andres Pena, David Fernando Romero, Sergio Raul Rivera Rodriguez, "Generation and Demand Scheduling in a Micro-grid with Battery-based Storage Systems, Hybrid Renewable Systems and Electric Vehicle Aggregators", WSEAS Transactions on Power Systems, pp. 8-23, Volume 14, 2019

[17]Lucjan Setlak, Rafal Kowalik, "The Dynamics of the Movement of Formations of Unmanned Aerial Vehicles", WSEAS Transactions on Applied and Theoretical Mechanics, pp. 192-197, Volume 14, 2019, Art. \#21,

[18] Lucjan Setlak, Rafal Kowalik, "The Dynamics of Group Flights of an Unmanned Aerial Vehicle" WSEAS Transactions on Applied and Theoretical Mechanics, pp. 129-139, Volume 14, 2019,

[19]Lucjan Setlak, Rafal Kowalik, "lgorithm Controlling the Autonomous Flight of an Unmanned Aerial Vehicle based on the Construction of a Glider", WSEAS Transactions on Applied and Theoretical Mechanics, pp. 56-65, Volume 14, 2019,

\section{Creative Commons Attribution License 4.0 (Attribution 4.0 International, CC BY 4.0)}

This article is published under the terms of the Creative Commons Attribution License 4.0 https://creativecommons.org/licenses/by/4.0/deed.en_US 